\title{
Análisis de la transfusión sanguínea alogénica en cirugía oncológica oral y maxilofacial. Análisis de variables
}

\author{
Analysis of allogeneic blood transfusion in oral and maxillofacial cancer \\ surgery. Analysis of variables \\ O. Altura Guillén' , A. Serrablo Requejo², I. Iso Rivera' ${ }^{1}$ V. Simón sanz'1, T. Rodríguez-Cachero', \\ E. Saura Fillat², I. Moral Saez², Uson Bouthelier², F. Hernández Altemir ${ }^{3}$
}

\begin{abstract}
Resumen: Objetivo. Analizar las variables que influyen en los pacientes transfundidos y sometidos a cirugía oncológica.

Material y método. Se han revisado y analizado los datos de 44 pacientes intervenidos quirúrgicamente de neoplasia, a los que se les ha practicado transfusión sanguínea peri operatoria, evaluando: edad, sexo, localización de la lesión, código de diagnostico, tratamiento realizado, hematocrito preoperatorio, hemoglobina preoperatoria y hemoglobina pretransfusional, numero de unidades transfundidas, estadio TNM, riesgo ASA y tiempo quirúrgico.

Resultados. 44 pacientes fueron transfundidos, 32 varones, con una media de edad de 65 años y con localización lingual en un 36,36\%. La cirugía mas frecuente fue la exéresis tumoral asociándose resecciòn ósea y vaciamiento cervical en el 56,82\% y con reconstrucción simple en el $63 \%$ de los casos. La duración media fue 5,7 horas, con un riesgo ASA medio de 3 y con una media de 2,9 unidades transfundidas. Un 70\% se encontraban en estadio IV. La $\mathrm{Hb}$ pretransfusional fue de media $7,71 \mathrm{~g} / \mathrm{dl}$. El tiempo quirúrgico, la $\mathrm{Hb}$ preoperatoria y el HTC preoperatorio son las variables que ha resultado estadísticamente significativas en el análisis multivariante.

Conclusiones. La transfusión sanguínea alogénica presenta una serie de efectos adversos que pueden condicionar la evolución del paciente oncológico, para evitar o disminuir estos efectos deletéreos se debe mantener criterios transfusionales restrictivos con $\mathrm{Hb}<8 \mathrm{~g} / \mathrm{dl}$, debemos actuar sobre la $\mathrm{Hb}$ preoperatoria, como única variable en la que podemos incidir para disminuir el volumen transfundido y promover programas de ahorro de hemoderivados.
\end{abstract}

Palabras clave: Cirugía oncológica; Anemia; Transfusión sanguínea alogénica; Autotransfusión.

Recibido: 09.06.2005

Aceptado: 16.06 .2008

\section{Médico Residente.}

2 Médico Adjunto.

3 Jefe de Servicio.

Servicio de Cirugía Oral y Maxilofacial.

Hospital Universitario Miguel Servet. Zaragoza, España.
Abstract: Objective. Analyze the variables that influence blood transfusion in patients undergoing surgery for cancer.

Material and method. Data from 44 patients who underwent surgery for neoplasms and required perioperative blood transfusion was analyzed to evaluate: age, sex, tumor location, diagnostic code, treatment, preoperative hematocrit, preoperative hemoglobin and pretransfusion hemoglobin, number of units transfused, TNM stage, ASA risk, and surgical time.

Results. Forty-four patients received transfusions (32 men). The mean age of patients was 65 years and the tumor was lingual in $36.36 \%$. The most frequent intervention was tumor exeresis, which was associated with bone resection and cervical lymph node clearance in $56.82 \%$ of cases and with simple reconstruction in $63 \%$ of cases. The mean duration of the intervention was 5.7 hours, mean ASA risk was 3, and mean transfusion volume was 2.9 units. Seventy percent of patients had stage IV tumors. Mean hemoglobin concentration before transfusion was $7.71 \mathrm{~g} / \mathrm{dl}$. Surgical time, preoperative hemoglobin concentration, and preoperative hematocrit were statistically significant in multivariate analysis.

Conclusions. Allogeneic blood transfusions originate adverse effects that can condition the evolution of patients with cancer. Strict transfusion criteria should be followed $(\mathrm{Hb}<8 \mathrm{~g} / \mathrm{dl})$ to prevent or diminish these deleterious effects. Preoperative $\mathrm{Hb}$ is the only variable that we can act on to reduce transfusion volume. Blood-sparing programs should be implemented.

Key words: Oncologic surgery; Anemia; Allogeneic blood transfusion; Autologous blood transfusion.

\section{Correspondencia:}

Dr. Oscar Miguel Altura Guillen

c/ Mayor 43, $2^{\circ}$ Izq, 50001-Zaragoza, España.

Email: oscaralt1@hotmail.com 


\section{Introducción}

En los últimos años se ha producido en los países desarrollados una disminución significativa de la utilización de sangre alogénica (TSA), y actualmente se están potenciando la investigación y la utilización de terapias alternativas, tales como la autotransfusión en sus diversas modalidades.

Esto es debido a la toma de conciencia de los riesgos que esta actitud terapéutica implica, ya que en realidad se trata de un transplante celular que somete al receptor a una sobrecarga de antígenos. Muchos de estos riesgos son ampliamente conocidos, como la posibilidad de transmisión de enfermedades infecciosas y los efectos adversos inmunológicos, como reacciones hemolíticas, febril, alérgica, anafiláctica, síndrome de distres respiratorio (SDRA), enfermedad de injerto contra huésped (EICH), púrpura postransfusional, etc. A estos hay que añadir la inmunomodulación inducida por la TSA (IMITA; transfusión-related immunomodulation: TRIM), que representa probablemente el efecto adverso mas preocupante en relación con la cirugía oncológica. ${ }^{1}$

Hoy en día no se conocen con exactitud cuales son los mecanismos que inducen esta inmunomodulación, pero si se sabe que esta implicada en numerosos efectos adversos que pueden condicionar la evolución del paciente oncológico sometido a transfusión sanguínea alogénica, tales como aumento de la infección nosocomial, aumento de la recidiva tumoral, ${ }^{2-3}$ e incluso como sugiere Taniguchi la transfusión de más de tres unidades de sangre alogénica provoca una disminución de la supervivencia. ${ }^{4}$

Es por esta serie de efectos adversos por lo que se deben iniciar programas de ahorro de hemoderivados, para lo cual debemos saber en primer lugar cuales son nuestros criterios transfusionales.

Para evaluar como son los criterios transfusionales en nuestro servicio, realizamos un estudio retrospectivo, analizando los factores que podrían influir a la hora de ser transfundidos los pacientes sometidos a cirugía oncológica del área oral y maxilofacial.

\section{Material y método}

Estudio retrospectivo de las variables que influyen en la transfusión sanguínea en los pacientes sometidos a cirugía oncológica.

Se han analizado las historias clínicas de aquellos pacientes sometidos a cirugía oncológica y transfusión sanguínea durante los últimos 18 meses ( 1 de Julio del 2002 al 31 diciembre del 2003) evaluándose la edad, el sexo, localización, estadio TNM, tiempo quirúrgico, riesgo ASA, Hg prequirúrgica, $\mathrm{Hg}$ y HTC pretransfusional, tipo de cirugía, tipo de reconstrucción y numero de unidades transfundidas. Posteriormente se establecieron las diferentes categorías de las distintas variables (Tabla 1).

Los datos se analizaron mediante el programa estadístico G-Stat, utilizándose el test de student en el análisis de la relación de las unidades transfundidas con las variables dicotómicas, el test de ANOVA un factor en el análisis de la relación de las unidades transfundidas con variables con mas de dos categorías, el test de correlación lineal para el análisis de la relación de las unidades transfundidas con la edad. Se realizó el test multivariante de regresión line-

\section{Introduction}

In recent years the use of allogeneic blood transfusions ( $A B T)$ has declined significantly in industrialized countries. The investigation and use of alternative therapies, such as different modalities of autologous transfusion, is flourishing.

Physicians are aware of the risks of allogeneic transfusions, which, in fact, are cellular transplantations that expose the transfusion receptor to an antigen overload. Many of the risks involved are well known, such as the possibility of transmitting infective diseases and immunologic adverse effects, including hemolytic, febrile, allergic, and anaphylactic reactions, respiratory distress syndrome (ARDS), graft-versushost disease (GVHD), post-transfusion purpura, and others. To these must be added transfusion-related immunomodulation (TRIM), which is probably the adverse effect that causes most concern in cancer surgery.

The mechanisms that induce transfusion-related immunomodulation are still not fully known, but immunomodulation is involved in numerous adverse effects that can condition the outcome of patients with cancer who undergo allogeneic blood transfusion. These include an increased incidence of nosocomial infection and tumor recurrence. 2,3 The transfusion of more than 3 units of allogeneic blood may reduce survival, as suggested by Taniguchi. ${ }^{4}$

Blood-sparing protocols should be initiated to prevent these adverse effects. However, transfusion criteria must first be clear in order to introduce blood-sparing strategies.

We evaluated the transfusion criteria of our department by means of a retrospective study of the factors that influence transfusion strategies in patients undergoing surgery for cancer of the oral and maxillofacial region.

\section{Material and method}

A retrospective study was made of the variables that influence blood transfusion in patients undergoing surgery for cancer.

The medical records of patients who underwent surgery for cancer and received blood transfusions during and 18month period (1 July 2002 to 31 December 2003) were analyzed. We evaluated patient age and sex, tumor location, TNM stage, surgical time, ASA (American Society of Anesthesiologists) risk, preoperative hemoglobin, pretransfusion hemoglobin and hematocrit, type of surgery, type of reconstruction, and number of units transfused. Variables were then categorized (Table 1).

Data were processed using the G-Stat statistical program. The Student test was used to analyze the relation between the number of units transfused and dichotomic variables. The relation between the number of units transfused and variables with more than two categories was examined by ANOVA. The relation between the number of units transfused and age was analyzed by linear correlation. A multi- 
Tabla 1. Variables y categorías.

\begin{tabular}{|c|c|}
\hline Variables y categorías & $\mathrm{N} / \%$ \\
\hline \multicolumn{2}{|l|}{ Sexo } \\
\hline Varón & $32(73 \%)$ \\
\hline Mujer & $12(27 \%)$ \\
\hline Edad (años) & $\begin{array}{r}\text { Media } \pm \text { DS } \\
65 \pm 14,4\end{array}$ \\
\hline \multicolumn{2}{|l|}{ Localización } \\
\hline Lengua & $16(36 \%)$ \\
\hline Suelo de boca & $5(11 \%)$ \\
\hline Maxilar & $4(9 \%)$ \\
\hline Encía & $4(9 \%)$ \\
\hline Labio & $4(9 \%)$ \\
\hline Orofaringe & $4(9 \%)$ \\
\hline Glándulas salivares & $3(6,8 \%)$ \\
\hline Retromolar & $2(4,5 \%)$ \\
\hline Yugal & $2(4,5 \%)$ \\
\hline \multicolumn{2}{|l|}{ Estadio } \\
\hline 1 & $0(0 \%)$ \\
\hline II & $7(16 \%)$ \\
\hline III & $8(14 \%)$ \\
\hline IV & $29(70 \%)$ \\
\hline Tiempo quirúrgico ( horas) & Media \pm DS \\
\hline Riesgo ASA & $5,7 \pm 1,3$ \\
\hline 1 & $0(0 \%)$ \\
\hline II & $12(27 \%)$ \\
\hline III & $28(64 \%)$ \\
\hline IV & $4(9 \%)$ \\
\hline V & $0(0 \%)$ \\
\hline Hb prequirúrgica (g/dl) & Media \pm DS \\
\hline Hombre & $13,7 \pm 2,3$ \\
\hline Mujer & $12,4 \pm 1,7$ \\
\hline Hb Pretransfusional (g/dl) & $\begin{array}{r}\text { Media } \pm \text { DS } \\
7,7 \pm 0,9\end{array}$ \\
\hline HTC pretransfusional (\%) & $\begin{array}{r}\text { Media } \pm \text { DS } \\
24,1 \pm 1,9\end{array}$ \\
\hline \multicolumn{2}{|l|}{ Tipo de cirugía } \\
\hline ERV & $25(57 \%)$ \\
\hline EV & $10(23 \%)$ \\
\hline ER & $9(20 \%)$ \\
\hline \multicolumn{2}{|l|}{ Tipo de reconstrucción } \\
\hline $\mathrm{S}$ & $28(64 \%)$ \\
\hline $\mathrm{C}$ & $16(36 \%)$ \\
\hline Unidades transfundidas & $\begin{array}{r}\text { Media } \pm \text { DS } \\
2,9 \pm 1,2\end{array}$ \\
\hline
\end{tabular}

al múltiple (Tabla 2) para el análisis de la relación de las unidades transfundidas con las variables cuantitativas.

\section{Resultados}

De los 44 pacientes sometidos a cirugía oncológica del área oral y maxilofacial y que fueron transfundidos durante un periodo de 18 meses ( 1 de Julio del 2003 al 31 diciembre del 2004) en el ser-
Table 1. Variables and categories.

\begin{tabular}{|c|c|}
\hline Variables and categories & $N / \%$ \\
\hline \multicolumn{2}{|l|}{$\operatorname{Sex}$} \\
\hline Men & $32(73 \%)$ \\
\hline Women & $12(27 \%)$ \\
\hline Age (years) & $\begin{array}{r}\text { Mean } \pm S D \\
65 \pm 14,4\end{array}$ \\
\hline \multicolumn{2}{|l|}{ Location } \\
\hline Tongue & $16(36 \%)$ \\
\hline Mouth floor & $5(11 \%)$ \\
\hline Maxilla & $4(9 \%)$ \\
\hline Gums & $4(9 \%)$ \\
\hline Lips & $4(9 \%)$ \\
\hline Oropharynx & 4 (9\%) \\
\hline Salivary glands & $3(6,8 \%)$ \\
\hline Retromolar & $2(4,5 \%)$ \\
\hline Cheek & $2(4,5 \%)$ \\
\hline \multicolumn{2}{|l|}{ Stage } \\
\hline 1 & $0(0 \%)$ \\
\hline II & $7(16 \%)$ \\
\hline III & $8(14 \%)$ \\
\hline IV & $29(70 \%)$ \\
\hline Surgical time (hours) & Mean $\pm S D$ \\
\hline ASA risk & $5,7 \pm 1,3$ \\
\hline I & $0(0 \%)$ \\
\hline II & $12(27 \%)$ \\
\hline III & $28(64 \%)$ \\
\hline IV & $4(9 \%)$ \\
\hline$V$ & $0(0 \%)$ \\
\hline Preoperative Hb (g/dl) & Mean $\pm S D$ \\
\hline Men & $13,7 \pm 2,3$ \\
\hline Women & $12,4 \pm 1,7$ \\
\hline Pretransfusion $\mathrm{Hb}(\mathrm{g} / \mathrm{dl})$ & $\begin{array}{r}\text { Mean } \pm S D \\
7,7 \pm 0,9\end{array}$ \\
\hline Pretransfusion Hct (\%) & $\begin{array}{r}\text { Mean } \pm S D \\
24,1 \pm 1,9\end{array}$ \\
\hline \multicolumn{2}{|l|}{ Type of surgery } \\
\hline ERV & $25(57 \%)$ \\
\hline$E V$ & $10(23 \%)$ \\
\hline$E R$ & $9(20 \%)$ \\
\hline \multicolumn{2}{|l|}{ Type of reconstruction } \\
\hline S & $28(64 \%)$ \\
\hline $\mathrm{C}$ & $16(36 \%)$ \\
\hline Units transfused & Mean $\pm S D$ \\
\hline
\end{tabular}

ERV: Tumor exeresis, bone resection, and cervical lymph node clearance; EV: Tumor exeresis and cervical lymph node clearance; ER: Tumor exeresis and bone resection; S: Simple; C: Complex; Hb: Hemoglobin; HTC: Hematocrit. 
vicio de cirugía oral y máxilofacial del hospital universitario Miguel Servet de Zaragoza, 32 eran varones y 12 mujeres, con una media de edad de 65 años con un rango de (29-88 años), la localización más frecuente fue la afectación lingual 16 casos, seguidos por la afectación del suelo de la boca 5 casos y con una frecuencia muy parecida para el resto de las localizaciones (Fig. 1). De los 44 pacientes 29 se encontraban en estadio IV (70\%), 8 en estadio III y 7 en estadio II. El tiempo medio del procedimiento quirúrgico fue de 5,7 horas $\pm 1,3$ con un rango de 2-8 horas, 28 pacientes (64\%) presentaban un riesgo ASA de III, 12 pacientes (27\%) presentaban un riesgo ASA de II y solamente 4 pacientes (9\%) presentaban un riesgo ASA de IV.

El procedimiento quirúrgico que con más frecuencia se realizó fue la exéresis tumoral asociándose resección ósea y vaciamiento cervical, no se distingue entre las diferentes modalidades de vaciamiento, realizándose en 25 pacientes (57\%), en 10 casos (23\%) se realizó una exéresis de la tumoración asociándose el vaciamiento cervical y en 9 casos (20\%) se realizó una exéresis de la lesión junto con una resección ósea. El tipo de reconstrucción que se realizo con más frecuencia fue una reconstrucción, simple 28 casos (64\%), entendiéndose por esta aquella que no precisó ningún tipo de colgajo para su reconstrucción, mientras que en 16 casos (36\%) se realizó una reconstrucción compleja, es decir aqueIla que preciso algún tipo de colgajo. La $\mathrm{Hg}$ prequirúrgica fue estratificada por el

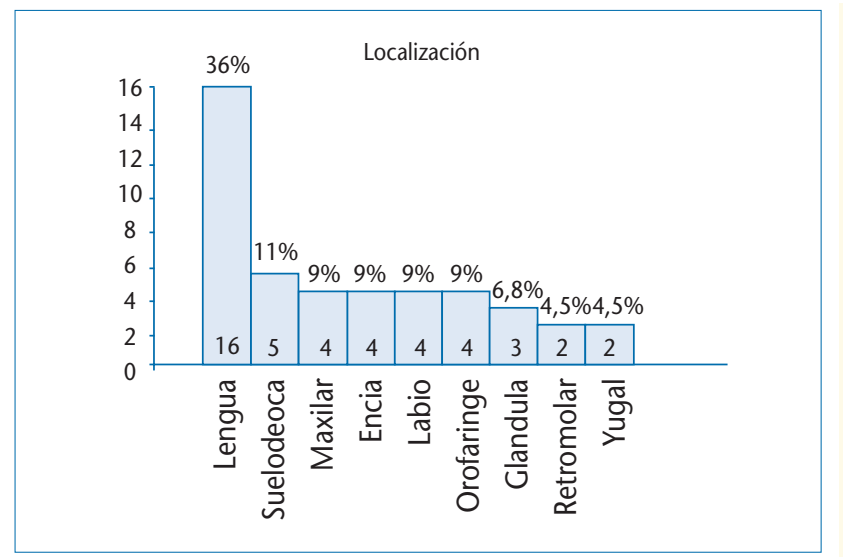

Figura 1. Localización.

Figure 1. Localization.
Tabla 2. Regresión lineal múltiple

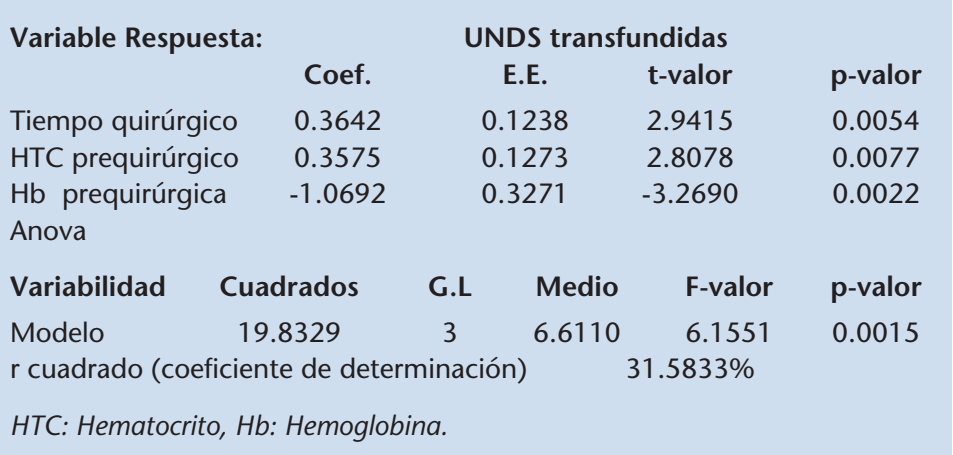

abla 2. Multiple linear regression
Response Variable:

Surgical time

Preoperative Hct

Preoperative $\mathrm{Hb}$

Anova

Variability

Model $19.8329 \quad 3$

squared (coefficient of determination)

Hct: Hematocrit; Hb: Hemoglobin. in an 18-month period (1 July 2003 to 31 December 2004) in the oral and maxillofacial surgery department of Miguel Servet University Hospital of Zaragoza. The patients included 32 men and 12 women; mean age was 65 years (range 29-88 years). The most frequent tumor location was the tongue (16 cases), followed by the floor of the mouth (5 cases) and other locations of similar frequency (Table 3). Of the 44 patients studied, 29 had stage IV disease (70\%), 8 had stage III disease, and 7 had stage II disease. The mean duration of the surgical procedure was $5.7 \pm$ 1.3 hours (range 2-8 hours). Twenty-eight patients (64\%) presented an ASA risk of III, 12 patients (27\%) ASA risk II, and only 4 patients (9\%) ASA risk IV.

The surgical procedure most often performed was tumor exeresis associated with bone resection and cervical lymph node clearance. The type of cervical lymph node clearance persexo, así en varones presentaron una media de 13,7 gr/dl $\pm 2,3$ y en mujeres una media de 12,4 $\pm 1,7$. La media de hemoglobina pretransfusional fue de $7,7 \pm 0,9$ y la media de hematocrito prequirúrgico fue de 24,1 $\pm 1,9$. La media de unidades transfundidas fue de 2,9 1,2 (Fig. 2).

No se hallaron diferencias estadísticamente significativas entre las unidades transfundidas y sexo, edad, estadio tumoral, procedimiento quirúrgico, y resección ósea.

Aunque no existen diferencias estadísticamente significativas, sí que existe cierta tendencia a una mayor transfusión en los casos de reconstrucción compleja $(p=0,0652)$ y de la misma forma existe una mayor tendencia a la transfusión en los casos de afectación del formed was not differentiated, but 25 patients (57\%) underwent this procedure. In 10 cases (23\%), tumor excision was associated with cervical lymph node clearance alone and in 9 cases (20\%), tumor excision was associated with bone resection alone. The reconstruction procedure was most frequently simple reconstruction, in 28 cases (64\%). This was understood to be reconstruction without mobilizing any skin flap. Complex reconstruction requiring some type of flap was performed in 16 cases (36\%). Preoperative hemoglobin concentration was stratified by sex: mean preoperative hemoglobin concentration in men was $13.7 \pm 2.3 \mathrm{~g} / \mathrm{dl}$ and in 
suelo de boca y maxilar superior $(p=$ $0,0545)$ con respecto a las demás localizaciones.

Se hallaron diferencias estadísticamente significativas, en el análisis multivariante aplicando el test de regresión lineal múltiple, al analizar el numero de unidades transfundidas y el tiempo quirúrgico $(p=0,0054)$ el HTC prequirúrgico $(p=0,0077)$ y la Hg prequirúrgica $(p=$ 0,0022).

\section{Discusión}

Desde que en los años 80 Francis y Sentón, ${ }^{5}$ demostraran que las transfusiones sanguíneas alogénicas estimulaban el desarrollo tumoral en ratas y Gantt, 6 sugiriera que las transfusiones alogénicas podrían ser beneficiosas para el receptor de un transplante renal al producir un estado de inmunosupresión y en consecuencia podrían tener un efecto adverso en los pacientes con cáncer, han sido muchos los autores que se han interesado por el tema, aunque es escasa la bibliografía en lo concerniente a la transfusión sanguínea alogénica en pacientes con neoplasias del ámbito de cabeza y cuello así como estudios randomizados sobre los efectos adversos que esta produce.

Es por todo esto por lo que se deben de poner en marcha mecanismos de ahorro de hemoderivados y actuar sobre las variables que influyen en el total de sangre transfundida.

En primer lugar debemos saber cuáles son los factores de riesgo para la transfusión sanguínea, así según V. Pinto García, ${ }^{7}$ éstos son fundamentalmente dos: en primer lugar el diagnóstico y en segundo lugar la técnica quirúrgica. En los pacientes con diagnóstico de neoplasia el riesgo relativo de transfusión es de 1 y según la técnica quirúrgica el riesgo de transfusión en cirugía ablativa de tejidos blandos es de 14,1, en la cirugía ablativa de los huesos es de 13,9 y en la cirugía reconstructiva de huesos es de 24,6.

Es importante saber cuáles son las variables sobre las que podemos actuar para intentar minimizar el volumen de sangre transfundida. Así en nuestro estudio estas son el HTC prequirúrgico, la Hb prequirúrgica y el tiempo quirúrgico. Para actuar sobre el tiempo quirúrgico se debe intentar que todos los procedimientos quirúrgicos sean realizados por equipos experimentados en cirugía oncológica y reconstructiva.

Se puede actuar sobre los niveles de hemoglobina y hematocrito prequirúrgico, valorando aquellos pacientes que presenten un estado anémico con una $\mathrm{Hb}<11,5 \mathrm{~g} / \mathrm{dl}$ en mujer y $\mathrm{Hg}<13 \mathrm{~g} / \mathrm{dl}$ en hombres, ya que según Okuyama. ${ }^{8}$ en estos pacientes anémicos, la simple suplementación con sulfato de hierro por vía oral durante dos semanas previas a la cirugía disminuye el riesgo de transfusión sanguínea de $27,7 \%$, en pacientes sin suplementación de hierro a 9,4\%.

Los valores hematológicos, a partir de los cuales, estaría indicada la transfusión sanguínea, deben ser lo mas restrictivos posibles, en nuestro estudio se ha utilizado un criterio transfusional restrictivo con una media de $\mathrm{Hb}$ pretransfusional de 7,7 g/dl $\pm 0,9$. women, $12.4 \pm 1.7 \mathrm{~g} / \mathrm{dl}$. Mean pretransfusion hemoglobin concentration was $7.7 \pm 0.9 \mathrm{~g} / \mathrm{dl}$ and mean preoperative hematocrit was $24.1 \pm 1.9 \%$. The mean transfusion volume was $2.9 \pm 1.2$ units (Table 4).

No statistically significant differences were found between the number of units transfused and sex, age, tumor stage, surgical procedure, or bone resection.

Nonetheless, a tendency to transfuse more blood was observed in cases of complex reconstruction ( $p=0.0652$ ). Likewise, there was a tendency to transfuse more blood in patients with tumors of the floor of the mouth and upper maxilla $(p=0.0545)$ than in tumors in other locations.

Statistically significant differences appeared in multivariate analysis when multiple linear regression was used to analyze the number of units transfused and surgical time ( $p$ $=0.0054)$, preoperative hematocrit $(p=0.0077)$, and preoperative hemoglobin $(P=0.0022)$.

\section{Discussion}

Francis and Sentón ${ }^{5}$ demonstrated in the 1980s that allogeneic blood transfusions stimulate tumor development in rats. Gantt6 proposed that allogeneic blood transfusions could benefit kidney transplant receptors by producing a state of immunosuppression but that this effect could be adverse for patients with cancer. Since then, many authors have studied the topic, although there is little bibliography on allogeneic blood transfusion in patients with neoplasms of the head and neck or randomized studies of the adverse effects of the procedure.

For this reason, blood-sparing protocols are needed and we should act on the variables that influence the total amount of blood transfused.

In first place, we need to know what factors make blood transfusion necessary. According to V. Pinto García,7 there are two fundamental risk factors: the first are related to the diagnosis and the second, to the surgical technique. Patients diagnosed of neoplasm have a relative risk of transfusion of 1. The risk of transfusion then depends on the surgical technique used, being 14.1 in ablative soft tissue surgery, 13.9 in ablative bone surgery, and 24.6 in reconstructive bone surgery.

It is important to know what variables we can try to act on to reduce transfusion requirements. Our study showed that these variables were preoperative hematocrit, preoper- 
Cuando encontramos valores de $\mathrm{Hb}$ entre 7 y $10 \mathrm{~g} / \mathrm{dl}$ la decisión de iniciar una transfusión sanguínea no debe ser tomada solamente por las cifras que muestren los valores hematológicos, sino por la situación clínica en la que el paciente se encuentra, ya que hay casos en los que los mecanismos compensatorios son suficientes para mantener las demandas metabólicas haciendo innecesaria la transfusión sanguínea. ${ }^{9}$

Debemos identificar aquellos pacientes en los que puede estar indicada la autodonación y promoverla. Se puede realizar un programa de autodonación cuando el riesgo de ser transfundido sea superior al 5\%,10 en nuestro caso presentamos una tasa de transfusión global del 33\%, con lo que estaría indicado la inclusión de nuestros enfermos en un programa de autotransfusión. Se debe comentar que existe una gran variabilidad con respecto a la utilización de las unidades obtenidas mediante el programa de autotransfusión, pudiendo existir una sobrevaloración de las necesidades transfusionales, en la literatura revisada encontramos que según algunos autores como Nath y Pogrel solo se utilizan un $24,2 \%$ de las unidades obtenidas, ${ }^{11}$ aunque también existen otros autores que refieren tasas de utilización del $50 \%$ y hasta del $73 \% .{ }^{12,13}$ Este modelo de ahorro de hemoderivados no solo disminuye los efectos adversos de las transfusiones alogénicas sino que también supone un ahorro en el coste. ${ }^{14}$

Existen otras estrategias de ahorro de hemoderivados como la hemodilución normovolémica, la cual puede evitar hasta un 89\% de las transfusiones alogenicas, ${ }^{15}$ la hipotensión controlada, ${ }^{16}$ la reinfusión de la sangre lavada y centrifugada desde el campo operatorio $^{17}$ y el uso de eritropoyetina. ${ }^{16,18}$

\section{Conclusiones}

La transfusión sanguínea alogénica perioperatoria produce una seria de efectos adversos que aumentan la morbi-mortalidad en los pacientes sometidos a cirugía oncológica. Para evitar o disminuir estos efectos deletéreos debemos promover políticas de ahorro de hemoderivados y actuar sobre las variables que influyen en el total transfundido, de esta forma la única variable sobre la que podemos incidir directamente es la hemoglobina preoperatoria, para esto se deben identificar aquellos pacientes anémicos e iniciar una suplementación con hierro oral. Además debemos mantener criterios transfusionales restrictivos transfundiendo con $\mathrm{Hg}<8 \mathrm{~g} / \mathrm{dl}$ si la situación clínica del paciente nos lo permite.

\section{Bibliografía}

1. Muñoz M, Llau V, Ramon S,Garcia JA. Transfusión sanguínea perioperatoria en el paciente neoplásico. I. Alteraciones inmunológicas y consecuencias clinicas. Cir Esp 2002; 72:160-8.

2. Mynster T, Chiristensen IJ, Moesgaard F, Nielsen HJ. Effects of the combination of blood transfusion and postoperative infectious complications on prognosis after surgery for colorectal. Br / Surg 2000;87:1553-62.

3. Moir MS, Samy RN, Hanasomo MM, Terris DJ. Autologous and heterologous blood transfusion in head and neck cancer surery. Arch Otolaryngol Head Neck Surg 1999;125:864-8. ative hemoglobin, and surgical time. Surgical time can be addressed by ensuring that all surgical procedures are performed by teams experienced in oncologic and reconstructive surgery.

Hemoglobin concentration and preoperative hematocrit can be addressed by evaluating patients to detect anemia ( $\mathrm{Hb}<11.5 \mathrm{~g} / \mathrm{dl}$ in women and $\mathrm{Hb}<13 \mathrm{~g} / \mathrm{dl}$ in men). According to Okuyama, 8 the administration of simple oral iron sulfate supplements to anemic patients for two weeks prior to surgery diminishes the risk of blood transfusion from $27.7 \%$ to $9.4 \%$ compared to patients with untreated anemia.

The hematologic values at which blood transfusion is indicated should be as strict as possible. In this study we applied a strict transfusion criterion of pretransfusion mean hemoglobin concentration $7.7 \pm 0.9 \mathrm{~g} / \mathrm{dl}$. Moreover, when the hemoglobin concentration is $7-10 \mathrm{~g} / \mathrm{dl}$, the decision to initiate blood transfusion is not taken solely on the basis of hematologic values, but also the patient's clinical situation. In some cases, compensatory mechanisms are sufficient to address metabolic demands, thus making blood transfusion unnecessary. ${ }^{9}$

We should identify patients from whom autologous blood can be collected and encourage the patient to do this. An autologous blood collection program can be initiated when the risk of transfusion is greater than $5 \% .10$ In our study, the overall transfusion rate was $33 \%$, so an autologous blood donation collection would have been indicated. It should be noted that the amount of blood from autologous extractions that is finally used varies widely. Transfusion needs may be overestimated. In the literature reviewed, Nath and Pogrel reported that only $24.2 \%$ of the units obtained are used, 11 although other authors reports rates of use of $50 \%$ and even $73 \%{ }^{12,13}$ Use of a blood-sparing model not only avoids the adverse effects of allogeneic transfusions but also reduces costs. ${ }^{14}$

Other blood-sparing strategies, such as normovolemic hemodilution, can reduce allogeneic transfusions by up to $89 \% .{ }^{15}$ Such blood-sparing measures include controlled hypotension, ${ }^{16}$ reinfusion of washed and centrifuged blood harvested from the operating field, ${ }^{17}$ and the administration of erythropoietin. ${ }^{16,18}$

\section{Conclusions}

Perioperative allogeneic blood transfusions are accompanied by a series of adverse effects that aggravate morbidity and mortality in patients undergoing surgery for cancer. Blood-sparing strategies should be used to prevent or reduce the deleterious effects of allogeneic transfusions. Likewise, we must act on any variables that can condition the need for blood transfusion. The only variable of this type on which we can act directly is preoperative hemoglobin. Anemic patients must be identified before surgery and oral iron supplements should be given. We also must adhere strictly 
4. Taniguchi Y, Okura M. Prognostic significance of perioperative blood transfusion in oral cavity squamous cell carcinoma. Head Neck 2003;25:931-6.

5. Francis DMA, Shenton BK. Blood transfusion and tumor growth: evidence from laboratory animals. Lancet 1981;2:871.

6. Gantt CL. Red Cells for cancer patients. Lancet 1981;2:363.

7. Pinto Garcia V, de Vicente Rodriguez JC, López Arranz JS. Análisis de la transfusión en la cirugía maxilofacial. Rev Esp Cirug Oral y Maxilofac 2000;24:171-5.

8. Okuyama M, Ikeda K, Shibata T, Tsukahara Y, kitada M, Shimano T. Preoperative iron supplementation and intraoperative transfusión during colorectal cancer. Surg Today 2005;35:36-40.

9. Van Der Linden P. Transfusión strategy. Eur J Anaesthesiol 2001;18:493-4.

10. Walker RD. Technical Manual. 10th ed. Arlington. American Association of Blood Banks 1990;518.

11. Nath A, Pogrel MA. Preoperative autologous blood donation for oral and maxillofacial surgery: an analysis of 913 patients. J Oral Maxillofac Surg 2005;63:347-9.

12. Weber RS. A model for predicting transfusion requirements in head and neck surgery. Laryngoscope 1995;105:1-17.

13. Savoia HF, Metz J, Maxwell EL, Hauesler MN, Mellor J, Kiely S. utilization of preoperative autologous blood donation in elective surgery. ANZ / Surg 2002;72: 557-60.

14. De Castro RM. Bloodless surgery: establishment of a program for the special needs of the Jehovah's witness community-the gynecologic surgery experince at a community hospital. Am J Obstet Gynecol 2000;182:251.

15. Habler O, Schwenzer K, Zimmer K, Prager M, koing U, Oppenrtieder K, Pape A, Steinkraus E, Reither A, Buchrot A, Zwissler B. Effects of standardied acute normovolemic hemodilution on intraoperative allogenic blood transfusion in patients undergoing major maxillofacial surgery. J Oral Maxillofac Surg 2004;33:467-75.

16. Rohling RG, Haers PE, Zimmermann AP, Schanz U, Marquetand R, Sailer HF. Multimodal strategy for reduction of homologous transfusions in cranio-maxillofacial surgery. J Oral Maxillofac Surg 1999;28:137-42.

17. Rohling RG, Zimmermann AP, Zellweger R, Schanz U. Transfusion of Washed and centrifuged shed RBCs during maxillofacial surgery Affects cytokine coincentrtions. Transfusion 2000;40:1352-6.

18. Christopoulou M, Derartinian H, Hatzidimitriou G, latrou I. Autologous blood transfusion in oral and maxillofacial surgery patients with the use of erythropoietint. Maxillofac Surg 2001;29:118-22. to transfusion criteria, transfusing only when hemoglobin concentration is below $8 \mathrm{~g} / \mathrm{dl}$ and only if the patient's clinical situation makes blood transfusion necessary. 